Reprod. Nutr. Dévelop., 1986, 26 (1 B), 381-382.

\title{
Variations de l'insulinémie en début de repas chez la vache en lactation \\ P. FAVERDIN
}

Station de Recherches sur la Vache laitière, I.N.R.A St Gilles, 35590 L'Hermitage, France.

Summary. Compared to direct feed access, a prefeeding period of $5 \mathrm{~min}$ with visual and olfactive feed presentation leads to a rapid and significant increase of glycemia and insulinemia in lactating cows. This suggests that reflex insulin discharge occurs at the onset of a meal in ruminants.

Chez les monogastriques, une sécrétion reflexe d'insuline en tout début de repas, précédant l'absorption des nutriments, a été décrite (Louis-Sylvestre et Le Magnen, 1983). Chez le mouton Basset (1974) a également observé une augmentation rapide de l'insulinémie dès les 5 à 10 premières minutes d'un grand repas, même au cours d'un repas fictif. Cette étude a donc été entreprise dans le but de caractériser plus finement la sécrétion d'insuline en début de repas et de savoir si des phénomènes de nature sensorielle (vue et odorat dans l'essai 1, goût et appétibilité du repas dans l'essai 2) étaient de nature à modifier la glycémie et l'insulinémie durant cette période.

Matériels et méthodes. Dans chaque expérience, 5 vaches Pie-Noires en lactation ont reçu successivement chacun des traitements. Dans le premier essai, l'effet de la présence et de l'absence d'une période de présentation des aliments pendant 5 min avant le début du repas a été étudié. Dans le second essai, la distribution d'un repas témoin, constitué par un mélange de $60 \%$ d'ensilage de maïs et de $40 \%$ d'aliments concentrés (ration habituelle), et celle d'un repas très appétible constitué par les aliments concentrés seuls, l'ensilage de maïs étant distribué 30 min après, ont été comparées.

Dans les 2 essais, les prélèvements sanguins ont été effectués en continu lors du repas du matin à l'aide d'un cathéter placé dans la veine jugulaire. La glycémie et l'insulinémie dans le plasma ont été déterminées toutes les minutes pendant 30 à $35 \mathrm{~min}$. Cette période de mesure était divisée en 3 phases : (1) une phase préexpérimentale (10 min dans l'essai 1, 5 min dans l'essai 2), (2) une phase de présentation du repas de 5 min pendant laquelle on distribuait et brassait la ration de l'animal dans son auge sans qu'il puisse consommer, sauf pour le traitement témoin de l'essai 1 pour lequel la phase pré-expérimentale était prolongée de $5 \mathrm{~min},(3)$ et une phase de 20 min correspondant au début du repas.

Résultats et discussion. Dans le premier essai, la présentation préalable de la ration a nettement modifié l'insulinémie et la glycémie (fig. 1). Chez les animaux stimulés par la présentation de la ration, ces deux paramètres ont tendance à diminuer lors de la présentation de la ration comparativement au témoin, mais surtout, augmentent beaucoup plus $(P<0,05)$ dès les premières minutes du repas. L'augmentation de la glycémie est plus rapide que celle de l'insuline, mais de faible amplitude $(5 \mathrm{mg} / 100 \mathrm{ml}$ ). Ces variations se retrouvent dans l'essai 2 . Cet 
accroissement de l'insulinémie est uniquement dû à une stimulation sensorielle, et non pas aux nutriments. Un contrôle nerveux du pancréas durant cette période apparaît vraisemblable (Rohner-Jeanrenaud et Jeanrenaud, 1983).
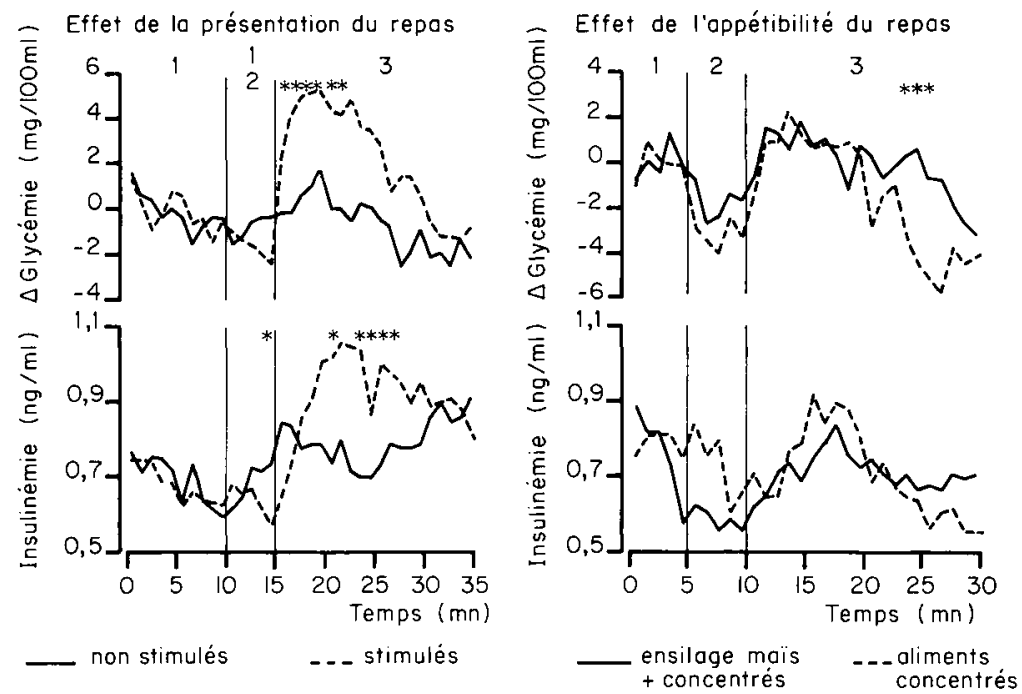

FIG. 1. - Variation de la glycémie et de l'insulinémie aux alentours de la distribution du repas chez la vache laitière. 1 = période pré-expérimentale, $2=$ présentation du repas, 3 = début du repas. * = différence significative à $P<0,05$ (Essai $1 n=5$, essai $2 n=4$ ). $\Delta$ Glycémie = glycémie - glycémie moyenne de la période de référence. E.T.M. = écart-type moyen d'un traitement pour chaque point de prélèvement.

Dans l'essai 2, la nature du repas n'a pas entraîné de modifications importantes de la glycémie et de l'insulinémie. En fait l'appétibilité des deux repas n'était pas très différente, l'ensilage de maïs étant de bonne qualité.

L'effet de la présentation du repas sur l'insulinémie a permis de montrer l'existence d'une phase " céphalique " de la sécrétion d'insuline chez la vache laitière dont les mécanismes restent à préciser.

Basset J. M., 1974. Early changes in plasma insulin and growth hormone levels after feeding in lambs and adult sheep. Aust. J. biol. Sci., 27, 157-166.

Louis-Sylvestre J., Le Magnen J., 1980. Palatability and preabsorptive insuline release. Neurosci. biobehavioral Rev., 4, 43-46.

Rohner-Jeanrenaud F., Jeanrenaud B., 1983. L'axe système nerveux central-pancréas endocrine. Ann. Endocr., 44, 217-227. 